\title{
Automatización dinámica de entornos documentales mediante tecnología workflow
}

\author{
Jesús González Lorca (1) \\ Fundación Séneca (Murcia)
}

\subsection{Resumen}

Se presentan las características, naturaleza y funciones más destacadas de la tecnología de flujos de trabajo, como alternativa para abordar los proyectos de automatización en entornos en los que el carácter documental es la nota predominante. Se ilustra con tres estudios de caso. La gestión de flujos de trabajo es un problema específico que no se puede resolver apropiadamente con aplicaciones documentales, sino complementarse con ellas.

Palabras clave: Flujos de trabajo. Automatización de procesos. Aplicaciones de trabajo en grupo. Coordinación. Gestión documental.

\section{2. Abstract}

The main characteristic and functions of workflow technology are presented as an alternative to automate processes in documentary environments, exemplified with three case studies. Managing workflows is an specific problem that cannot be properly solved with information retrieval software, but with a complementary approach.

Keywords: Workflow. Processes automation. Groupware. Coordination. Document management.

\section{Introducción}

Las organizaciones que tradicionalmente trabajan con la información como elemento esencial - esto es, entornos documentales - pueden calificarse como oficinas, término que engloba a aquellos entes que generan información, y por tanto documentación. Estas oficinas, al igual que el resto de tipologías organizativas, no están exenta de un carácter complejo, dado el entorno al que deben adaptarse, la gran cantidad de documentación con la trabajan y las necesidades de gestión que presentan. Los sistemas de gestión documental han sido la primera opción aplicada para la automatización de la documentación en estos entornos. 
Esas aplicaciones han vivido un verdadero auge en las organizaciones, sobre todo por el carácter eminentemente documental de una gran variedad de procesos. El problema es que se han empleado con el objetivo principal de la gestión de los procesos, no para lo que fueron realmente concebidas: la gestión documental, el tratamiento de la información.

Uno de los principales inconvenientes a los que se han enfrentado los sistemas de gestión documental, y razón de su fracaso como gestor de procesos, es el de la incapacidad de efectuar labores de coordinación e integración entre los elementos que participan en los procesos y que pertenecen a un entorno colaborativo. En realidad estos conceptos pertenecen al terreno de los sistemas de automatización de flujos de trabajo. Esta tecnología es la apropiada para abordar tales retos, pues no en vano se engloba dentro de las herramientas groupware, las cuales giran en torno a los ejes de acción de la comunicación, colaboración y coordinación, materializada a través de la mencionada automatización de flujos de trabajo.

La utilización de tecnología de flujo de trabajo produce en la organización un cambio en su orgánica, propiciando una forma de operar basada en los procesos en lugar de la errónea visión basada en las funciones. Así, se obtiene un modelo de organización centrado en los procesos que va a generar beneficios directos sobre éstos y sobre la documentación, que es lo verdaderamente interesante para estos entornos de oficina.

Los sistemas de automatización de flujos de trabajo permiten establecer una definición automatizada del proceso (flujograma) en la que se describen las actividades que los constituyen, los usuarios que intervienen, los recursos que van a emplear para resolverlas y las reglas que han de seguir en el procedimiento. Una vez se ejecuta el flujograma, el sistema envía automáticamente a cada usuario sus actividades, los recursos para cumplimentarlas y la documentación necesaria. De esta forma, se aporta al entorno un carácter dinámico, pues es la información la que acude a los usuarios, que solamente se preocupan por realizar las actividades bajo las condiciones establecidas.

\section{Complejidad de los entornos documentales}

\subsection{La oficina como productora de documentación}

Prácticamente la totalidad de las organizaciones utilizan los documentos como soporte de las actividades que desarrollan, aunque existe un tipo de entidades que, por su naturaleza y características, tienen en los documentos un elementos esencial, ya que constituyen tanto el soporte de sus procesos como también el resultado de los mismos; es decir, nueva documentación, que se traduce en servicios y/o productos. Se trata de los entornos documentales, organizaciones vinculadas estrechamente a los documentos a niveles de proceso y producto/servicio, como es el caso de la Administración Pública, las bibliotecas, los archivos y los 
centros de documentación, entre otros. Estos entornos u organizaciones pueden englobarse bajo el concepto de oficina, pero no utilizándolo desde el punto de vista de la industria o la producción, sino entendiendo oficina como una entidad de carácter administrativo o documental, pues su razón de ser son los documentos, los cuales constituyen la base de sus procesos. Sáez (1990), entre otros, emplea el término oficina considerando que es mucho más apropiado para hacer referencia al flujo documental y de actividades que forman los procesos.

La visión de la organización como oficina es la que más se acerca a su dimensión interior, a su funcionamiento y a las actividades que desarrolla. Esta visión constituye el epicentro del trabajo, pues la atención se fija en la aplicación de las tecnologías de la información y comunicación al entorno de la oficina vista como organización (González, 2001). Por otro lado, emplear este concepto será de utilidad para aportar, posteriormente, algo de luz en la complejidad del trabajo de las organizaciones y en la aplicación de la tecnología necesaria para automatizarlo de forma eficiente.

Sáez (1990) utiliza oficina como concepto que hace referencia a una organización a través de un modelo de complejidad que representa precisamente el trabajo que tiene lugar en una oficina, dividido en varios niveles - actividades (procesos individuales), grupos (proceso sistémico) y sistema social (proceso global)-, al que posteriormente le aplica sus correspondientes niveles de tecnología para su automatización (Caja de Herramientas-Sistema Tecnológico Ofimático-Sistema Ofimático) (figura 1). Se trata, por tanto, de un entorno estrechamente vinculado a lo que se supone es una organización, lo que valida su aplicación en el contexto de este trabajo.

Laudon y Laudon (1996) afirman que las oficinas son grupos compuestos de personas que cooperan para lograr unos objetivos comunes. De hecho, sostienen que sus tres papeles críticos en el seno de la organización son los siguientes:

1. Coordinan y administran el trabajo de los profesionales y trabajadores de la información dentro de la organización. En ésta, existen grupos o departamentos establecidos que desarrollan una actividad concreta (por ejemplo, finanzas, producción, etc.), cuyo trabajo es coordinado para alcanzar una mayor eficiencia en la gestión de dichas actividades departamentales.

2. Enlazan el trabajo que se realiza en todos los niveles y funciones de la organización. Los grupos, departamentos o áreas de trabajo son enlaza-

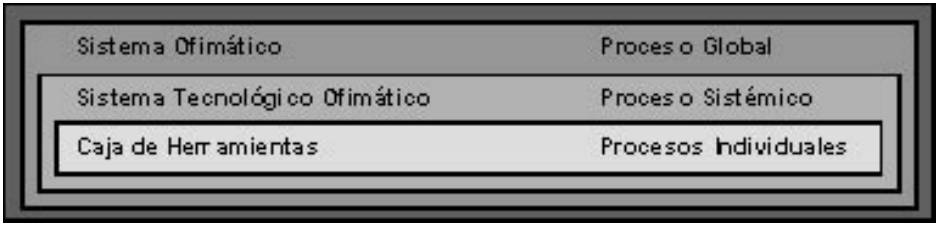

Fig. 1. Representación del modelo de tres niveles de complejidad (Sáez Vacas, 1990).

Scire. $9: 2$ (jul.-dic. 2003) 61-83. 
dos para establecer un flujo de trabajo continuo y dinámico para cumplir los objetivos de la organización.

3. Acoplan la organización al medio ambiente externo, incluyendo a sus clientes y proveedores. La actividad de la organización se pone en contacto con el entorno social externo para dar soporte a funciones o acciones concretas.

Se pone de manifiesto la importancia que adoptan los conceptos de coordinación e integración en el entorno de las oficinas y, por tanto, en el marco de la gestión de los documentos que dan soporte a los procesos de la organización.

\subsection{Problemática derivada de la gestión de documentación}

Cleveland (1995) sostiene que alrededor de un 90\% de la información que maneja una organización reside en los documentos, fijada manualmente. De esta forma, se impone el empleo de adecuadas técnicas de gestión de la documentación con el fin de que ésta sirva y responda de manera eficiente a los objetivos de la organización en la que fluye. El manejo de los documentos puede acarrear grandes y graves inconvenientes, sobre todo si se trata de un volumen considerable. Los problemas más comunes derivados de la gestión de documentos en una organización, son los siguientes (Ticap, 1999, p. 139):

- Dificultad de acceso: La documentación suele guardarse en archivos que pueden no estar próximos al lugar de trabajo, lo que produce retrasos en su entrega, debido a su búsqueda (siempre que los criterios de recuperación sean los correctos) y distribución.

- Indisponibilidad: Puede ocurrir que al solicitar una determinada documentación, ya esté siendo utilizada por otro usuario.

- Deterioro: La documentación en formato papel tiene el inconveniente de deteriorase por su continua utilización, deficiente almacenamiento o negligencias en su manejo, lo que ocasiona que con el tiempo pierda su calidad original.

- Pérdida: La documentación puede perderse al traspapelarse con otros documentos que utilizamos, o porque se coloque en lugar erróneo al devolverla al archivo, con el inconveniente de tener que volver a restablecerla.

- Redundancia: Con el uso continuo de documentación es habitual que se hagan copias de ésta para que la utilicen varias personas, que al final terminan siendo también archivadas, provocando el aumento del archivo.

- Dispersión: En la práctica, cada departamento de la organización termina creando su propio archivo para gestionar la documentación que utiliza, por lo que tendremos dificultades al consultar información de otras áreas, al tener un diferente tratamiento. 
- Seguridad: Si se desea establecer algún tipo de control de accesos en los archivos en papel, todo queda delegado a la persona que custodia el archivo, por lo que la seguridad será cuestionable.

- Espacio físico: La documentación en papel requiere archivos en locales de grandes dimensiones, pues con el tiempo su volumen será considerable.

- Costes de personal: Precisamente, el aumento de volumen de la documentación y sus archivos hace que se necesite más personal dedicado a su gestión y mantenimiento, aumentando así también su coste.

A este respecto, Saorín (1997) sostiene que la función de documentación en la organización está atrofiada, haciendo referencia a la incapacidad de los sistemas de documentación para la recuperación y consulta efectivas, lo que desemboca en la dependencia continua del formato papel, pérdida de datos y redundancia de operaciones; algo que, con el desarrollo tecnológico actual, no es tolerable. Siminiani afirma a este respecto, corroborando esta idea, que "la gestión manual de los documentos es normalmente muy ineficaz (mal servicio) e ineficiente (costes excesivos)" (Siminiani, 1997).

Como antes se ha mencionado, se impone la implantación de tecnologías que permitan una adecuada gestión de la documentación de forma automatizada. Siminiani la define como "gestión que tiene como objetivo proporcionar apoyo a la utilización de los documentos para cualquier tipo de proceso en cualquier entorno de la empresa, individual, departamental o corporativo" (Siminiani, 1997).

\section{Automatización de la gestión de documentos}

\subsection{Sistemas automatizados para la gestión documental}

Cuando se hace referencia al término gestión documental suele utilizarse en el sentido de la gestión o control automatizado de un conjunto de documentos electrónicos durante todo su ciclo de vida en una organización, desde que se inicia el proceso con su creación hasta que concluye con su almacenamiento definitivo (Cleveland, 1995). Este tipo de concepciones enfatizan el proceso que sufren los documentos en detrimento de lo que constituye la manipulación automatizada, que debe ser la idea predominante cuando se le otorga significado a tal término. De este modo, al hacer referencia en este trabajo a la gestión documental se opta por considerarla como el tratamiento de la documentación de una organización por medio de elementos electrónicos; es decir, el manejo automatizado de los documentos.

A menudo también se vincula el significado de gestión documental al de gestión del conocimiento, argumentando para ello que la información de la organización, que supone el conocimiento de la misma, se plasma en los documentos que fluyen por ella. Así, ambos conceptos resultan relacionados (Ticap, 1999).

Scire. $9: 2$ (jul.-dic. 2003) 61-83. 
Resulta vital para la organización, por tanto, controlar los flujos de conocimiento a través de la automatización de sistemas. Esta automatización se ha realizado en el seno de las organizaciones con el objetivo de alcanzar la ansiada oficina sin papeles (paperless office), aunque en realidad se ha llegado a la oficina con menos papeles (less paper office) (Calzado, 1996), que se asemeja más a la situación que actualmente presentan la mayoría de los entornos documentales (oficinas).

El Sistema de Gestión Documental (SGD) supone la herramienta tecnológica utilizada para la automatización de entornos documentales en primera instancia, por sus características, objetivos y prestaciones. Codina (1994) define un SGD como "un sistema que permite la automatización, creación, mantenimiento y consulta de fuentes de información constituidas por documentos y, por lo tanto, sirve para explotar el conocimiento que contienen los documentos con el fin de ponerlo al alcance de los usuarios del sistema". Al tratar lo conceptos de información y conocimiento se amplía el espectro de significación y comprensión de lo que supone un documento, considerando como tal toda información fijada en cualquier tipo de soporte: papel, magnético, electrónico e incluso el conocimiento adquirido de las personas.

El crecimiento desmesurado de la documentación y las necesidades derivadas de su tratamiento han producido el auge de los SGD en las organizaciones, ya sean de carácter público o privado. Su implantación viene dada por la obligación de controlar e incrementar la eficiencia del flujo de documentos que suponen la base en la que se sustentan los procesos de los entornos documentales u oficinas (Martínez e Hilera, 1997). Automatizar la gestión de documentos genera una serie de beneficios, entre los que cabe destacar el aprovechamiento del capital intelectual de la organización, ya que el conocimiento se crea una sola vez y se reutiliza muchas; el trabajo en equipo más efectivo acelerando actividades críticas para la organización, como por ejemplo la distribución; la mejora del proceso de producción (de documentos, en este caso) y del servicio al cliente, ya que el acceso a los documentos es inmediato; y la rapidez de respuesta frente a posibles eventos o imprevistos. Las organizaciones tienden a servirse de los SGD para optimizar sus procesos, sobre todo cuando se trata de procesos exclusivamente documentales; es decir, aquéllos cuya razón de ser son los documentos que lo componen y que son su causa y efecto. Su intención es reducir el ciclo de tales procesos y sus costes a la vez que incrementar su calidad (Calzado, 1999).

El inconveniente que presentan los SGD cuando se emplean con el fin de mejorar los procesos (que está más allá de sus propias limitaciones y de la razón por la que se crearon) es su carácter estático; es decir, la incapacidad de mover la información a través del sistema por ellos mismos. Posibilitan el establecimiento de modelos de datos para gestionar los documentos, pero éstos permanecen estáticos en el sistema a la espera de que los usuarios efectúen las búsquedas per-

Scire. $9: 2$ (jul.-dic. 2003) 61-83. 
tinentes que activen la localización y recuperación de la información solicitada. Los usuarios son los que deben acudir al encuentro de la documentación, cuando lo ideal y deseable es, como afirma Calzado (1996), que los SGD evolucionen hacia sistemas en los que la información sea la que acuda en busca del usuario, proporcionándole los documentos necesarios para efectuar las actividades que tiene encomendadas en el proceso en el que interviene.

\subsection{La necesidad de automatizar el flujo de trabajo en la gestión de los procesos}

Uno de los puntos negativos que presentan las organizaciones es su orgánica funcional. Esto quiere decir que su configuración se basa en la gestión de funciones, que persigue la eficiencia de los trabajadores, cuando en realidad debería establecerse una orgánica horizontal, cuyo objetivo es la eficiencia de los procesos (Siminiani, 1996). Este hecho es el que ha derivado en la utilización de sistemas de gestión documental para resolver el problema del tratamiento de la documentación de los procesos. Pero estos sistemas no están preparados para abordar la gestión de procesos, y menos aún automatizando entornos documentales sin tener en cuenta la importancia de la integración de sistemas, ya que la automatización se ha llevado a cabo por áreas o departamentos, lo que dificulta la coordinación y coherencia en lo referente a la información de los procesos. En los entornos corporativos es necesaria la coordinación de todos los elementos que forman parte de sus procesos, lo que incluye la documentación que los sustenta.

La acción de automatizar la gestión documental no hace sino provocar un espejismo sobre el vasto desierto de documentos, ya que no va a lograr la supresión de los problemas que la documentación lleva arraigados, que es la creencia habitual. La realidad a la que debe enfrentarse la organización es la de aplicar tecnologías adecuadas que permitan llevar a cabo una gestión eficiente y efectiva de su información. La causa más común de la situación de la gestión de documentación viene provocada por la falta de integración en los sistemas automatizados. Lo cierto es que, dada la gran relevancia que tiene la información para la organización, es de vital importancia establecer mecanismos que lleven a la integración y coordinación de los flujos de información y de actividades que tengan que ver con esa información; esto es, los procesos informativos de la organización.

Si se aplica una gestión documental automatizada se requiere un complemento tecnológico que suprima el carácter estático del sistema y lo sustituya por uno dinámico, con el que los documentos sean capaces de fluir por el proceso, buscando al usuario pertinente que los necesita para desarrollar las actividades asignadas. Ese carácter dinámico, a la vez que integrador y coordinador, lo aporta la tecnología de automatización de flujos de trabajo (workflow). Esta colaboración tecnológica aporta una gran ventaja a la gestión de los procesos, ya que "cuando un gestor recibe la indicación de realizar una actividad, puede recibir simultánea-

Scire. $9: 2$ (jul.-dic. 2003) 61-83. 
mente los documentos a cumplimentar, los ya cumplimentados, los documentos que tienen las instrucciones para cumplimentarlos, el historial, la jurisprudencia, etc." (Siminiani, 1996). Todo esto viene a significar que el proceso se gestiona de forma eficiente, pues los usuarios que intervienen están totalmente documentados y preparados para llevar a cabo su cometido.

\section{Sistemas de automatización de flujos de trabajo}

\subsection{Vinculación con la tecnología groupware}

El objetivo final de las Tecnologías de la Información y Comunicación (TIC), y concretamente del groupware, es el de proporcionar un soporte activo a las funciones y al trabajo que se desarrollan en las oficinas donde se aplican, permitiendo el establecimiento y configuración de grupos capaces de interaccionar. Esta tecnología se encuadra dentro de los denominados sistemas de automatización de oficinas, aplicaciones que pretenden el incremento de la productividad de los trabajadores de la información que integran la oficina (Laudon y Laudon, 1996).

El groupware es una tecnología orientada a facilitar el trabajo conjunto de grupos de usuarios creando un entorno colaborativo. Sagredo lo define como "herramientas con las que las personas pueden trabajar juntas en un marco colectivo de comunicación, colaboración y coordinación" (Sagredo, 1996). Existe una gran controversia acerca de lo que se entiende por groupware, por lo que existen numerosas definiciones. La primera y original que se le atribuyó al groupware data de 1981, siendo su autoría de Peter y Trudy Johnson-Lenz, los cuales lo concibieron como "GROUP proceses and plus softWARE support", lo que viene a significar "procesos para desarrollar en grupo y su software de soporte" (JohnsonLenz, 1982). Su funcionamiento se basa en tres ejes (figura 2), los cuales representan la forma en que los usuarios trabajan al formar grupos (Ultimus, 1998):

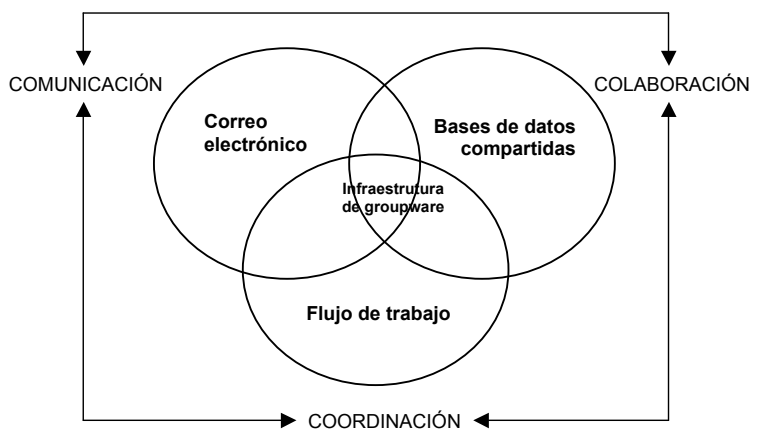

Fig. 2. Esquema representativo del trabajo en grupo (Sagredo, 1996).

Scire. $9: 2$ (jul.-dic. 2003) 61-83. 
a. Comunicación, a través del envío de información. Se basa en la utilización de tecnología de mensajería electrónica.

b. Colaboración, efectuada al compartir la información. Tiene su fundamento en el establecimiento de espacios virtuales para compartir la información y la utilización de bases de datos compartidas.

c. Coordinación, al integrar todas las funciones en un único entorno que propicia un desarrollo efectivo de los procesos. Es una función vital para el éxito de los procesos.

Al tratarse la coordinación de una función que engloba todos los ejes de acción del groupware, además de erigirse como la razón de ser de la tecnología de flujo de trabajo, es de rigor detallar los aspectos que la caracterizan. La coordinación permite la adaptación de la oficina a las nuevas necesidades de información, pues ésta constituye un entorno cambiante. La coordinación implica la existencia de diversas funciones en el marco de la oficina, las cuales dan lugar a las actividades que forman parte de los procesos. Es una función orientada al proceso, por lo que las actividades han de estar definidas correctamente, aunque cada organización lleva a cabo la coordinación según las características de sus propios procesos, no en función de un modelo establecido o normalizado de actuación (González, 2001). El ámbito de la coordinación es el campo donde actúan con eficacia los sistemas de automatización de flujos de trabajo (workflow systems), sistemas que llevan a cabo la definición, ejecución y gestión de procesos de una forma automatizada. Son de utilidad para el trabajo de oficina al aportar dinamismo e integración.

En definitiva, la finalidad de incorporar en el groupware la capacidad de coordinar es la de "permitir a las organizaciones tener control e incrementar la eficiencia de los flujos de documentos que soportan sus negocios o actividades" (Martínez e Hilera, 1997), lo que corrobora la vinculación - y, al mismo tiempo, validez de aplicación - de esta tecnología a los entornos documentales, o lo que es lo mismo, al trabajo de oficina.

\subsection{Concepto y definición de automatización de flujos de trabajo}

Al trabajar con herramientas de automatización de flujos de trabajo se está utilizando una tecnología de control y coordinación, pues su objetivo es la automatización de la secuencia o flujo de actividades de un proceso y su distribución a los usuarios junto a los recursos que necesitan (aplicaciones, documentos, etc.) para su cumplimiento, en función de un conjunto establecido de reglas.

Su finalidad se entiende perfectamente, aunque esta unanimidad no está presente a la hora de definir el término. Como afirma Lee Thé, "si se pide a 10 personas que definan flujo de trabajo, se obtendrán 11 respuestas distintas" (Thé, 1995); lo que da cuenta de la dificultad que entraña establecer una definición

Scire. $9: 2$ (jul.-dic. 2003) 61-83. 
al respecto. A pesar del colapso y la confusión existente, la WfMC (Workflow Management Coalition o Coalición para la Gestión del Flujo de Trabajo), organización internacional no lucrativa formada por usuarios, distribuidores y analistas de flujo de trabajo, y cuya finalidad es la normalización de terminología, conectividad e interoperatibilidad de estos sistemas, establece una definición, a través de su Modelo de Referencia del Flujo de Trabajo (Hollingsworth, 1995), en la que considera que se trata de "la automatización de un proceso de negocio, total o parcialmente, en el que información de todo tipo llega al usuario adecuado en el momento adecuado, en base a un conjunto de reglas inteligentes, que permite que la mayoría del trabajo sea efectuado informáticamente, mientras que las personas se ocupan solamente de las excepciones" (WAC, 1998).

\subsection{Evolución de flujos manuales a flujos automatizados}

Como anteriormente se ha comentado, el hecho de que los procesos de la organización se caractericen por su alta carga documental obliga a que éstos se desarrollen de forma manual, a pesar de los intentos por automatizarlos mediante SGD. Esta contrariedad impide dar dinamismo al flujo de documentos, ya que no existe una clara integración de todos los elementos participantes en los procesos, además de que su heterogeneidad tampoco facilita las cosas. La falta de integración origina una serie de problemas en el marco de los procesos, entre los que cabe destacar cinco (Hales, 1998): la gran dependencia del papel, al no poder automatizar todos sus elementos constituyentes; la pérdida de documentos, dado su alto grado de utilización; la fragmentación de las actividades; la dificultad de efectuar el seguimiento activo del estado de las actividades por la dispersión de los datos; y las pocas inversiones en TIC, debido a la alta dependencia del papel, actividades manuales y recursos humanos.

Es lógico pensar, por tanto, que para la organización se impone el hecho de afrontar esta situación generando un nuevo punto de vista en la metodología de su trabajo, aunque si bien se suelen mostrar un tanto recelosas y cautas a la hora de introducir modificaciones en el entramado de su estructura. Una de las soluciones más lógicas y ventajosas supone la aplicación de tecnologías de automatización del flujo de trabajo, debido a que son capaces de integrar en un mismo entorno todo el cúmulo de componentes heterogéneos que participan en el trabajo (WAC, 1998). ¿Qué va a aportar esta tecnología a la eficiencia de los procesos? Estas prestaciones pueden resumirse en las siete siguientes: integración de los componentes que intervienen en los procesos, independencia entre la lógica del proceso y el hardware y software de soporte, creación, modificación y gestión de flujos automatizados de procesos, garantía de que la información llega al usuario pertinente en el momento adecuado, monitorización (seguimiento) y reasignación (cuando sea necesario) de las actividades, integración de aplicaciones y hardware hetero-

Scire. $9: 2$ (jul.-dic. 2003) 61-83. 


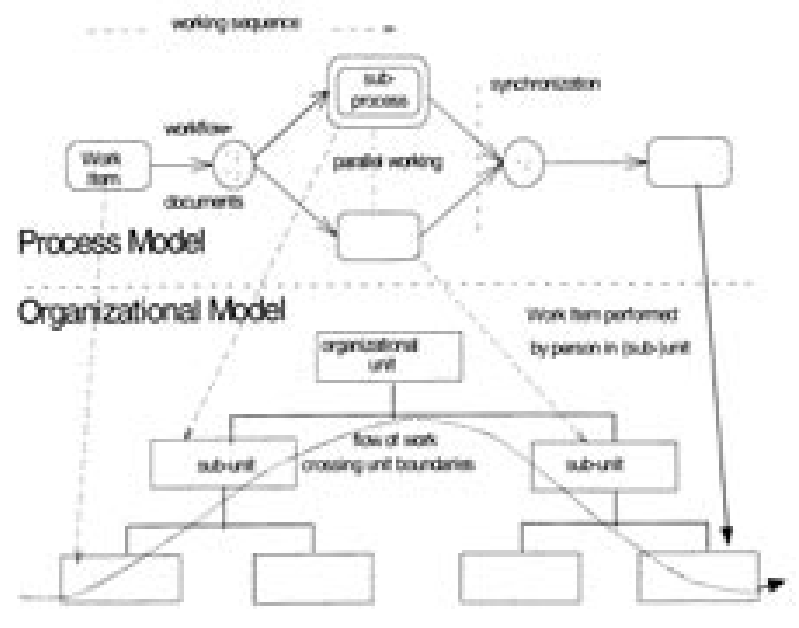

Fig. 3. Abstracción de un modelo de proceso de la organización (Vlachantonis, 1998). géneos, con garantía de compatibilidad, y procesamiento del trabajo en función de reglas establecidas sobre su cumplimiento.

La automatización de flujos de trabajo tiene como cualidad principal la capacidad de integrar, como se ha mencionado, los sistemas existentes e implicados en el proceso. En palabras de Pyke "el flujo de trabajo es el pegamento que une sistemas y entornos [...] esta integración ha de cubrir todos los aspectos de los procesos de negocio" (Pyke, 1998). Esta característica constituye para la organización un primer paso hacia la conversión de su orgánica funcional en una orgánica basada en los procesos, lo que la capacita para afrontar el desarrollo de nuevas estructuras y sistemas de información, beneficiando, de esta forma, a sus productos y servicios, como afirma Vlachantonis (1998). Este autor sostiene la tesis de que con una estructura basada en funciones el individuo ve limitada su percepción de la realidad organizativa, pues solo se preocupa del trabajo que desarrolla en su unidad o departamento. $\mathrm{Al}$ encontrarse esclavo de una jerarquía, no concibe la organización como algo global. Esto provoca una clara dificultad en la comunicación entre unidades e individuos, además de la ralentización del trabajo desarrollado. La orientación hacia los procesos horizontaliza la estructura organizativa (figura 3), estableciendo un marco de integración, comunicación y colaboración entre los elementos de los procesos. El hecho de que cada elemento del flujo del proceso se vincule con componentes de la estructura de la organización genera una visión horizontal del trabajo que se superpone al esquema de funcionamiento de la organización.

\subsection{Esquema de funcionamiento de un sistema genérico}

La Coalición para la Gestión del Flujo de Trabajo (WfMC) ha establecido un Modelo de Referencia para el Flujo de Trabajo (Hollingsworth, 1995) con el fin de proponer un sistema estándar formado por una serie de módulos o interfases que representan las funciones características de la automatización de flujos, a saber:

Scire. $9: 2$ (jul.-dic. 2003) 61-83. 
- Interfaz 1: Definición de procesos.

- Interfaz 2: Aplicaciones cliente.

- Interfaz 3: Aplicaciones invocadas.

- Interfaz 4: Interoperatibilidad con otros sistemas de flujo de trabajo.

- Interfaz 5: Herramientas de Administración y monitorización.

- Entorno de generación del flujo de trabajo.

El Modelo de Referencia permite que coexistan en un mismo entorno diferentes productos de flujo de trabajo gracias a la normalización de las especificaciones de los diversos módulos. Así, una organización puede utilizar un módulo de definición de un productor diferente al de su módulo de administración, etc.

Esta normalización ha sido posible gracias, en primer lugar, a los esfuerzos por analizar los diferentes productos de flujo de trabajo. Se llegó a la conclusión de que, pese a su heterogeneidad, poseen un conjunto de características y funciones comunes. De esta forma, se estableció un modelo común o genérico que contiene todos estos elementos (figura 4), que además es de utilidad para explicar el funcionamiento de estas herramientas. La mayoría de los productores aceptan y emplean este modelo genérico para la automatización de

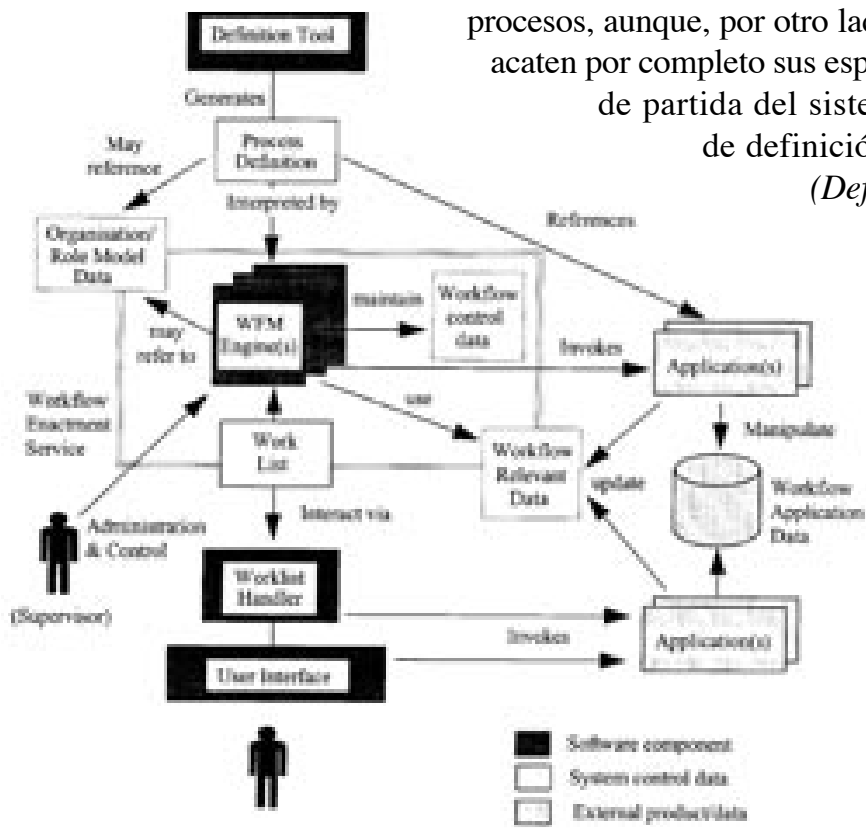

Fig. 4. Estructura genérica de un sistema de flujo de trabajo (Hollingsworth, 1995)
(Definition Tool), mediante la cual se diseñan los flujos normalizados de los procesos a automatizar (Process Definition). Esta definición del proceso puede estar vinculada a un mapa de la organización basado en roles (Organisation/Role Model Data) para asignar actividades a dichos roles o, en su defecto, vincularse directamente con los usuarios del 
flujo. Además, se establece un enlace entre las actividades y las aplicaciones informáticas (Applications) necesarias para su cumplimiento. Una vez se ha realizado la definición del flujo, ésta pasa al entorno de generación del flujo de trabajo (Workflow Enactment Service), un marco de trabajo donde se interpreta la definición del flujo de trabajo y se controla su ejecución mediante su componente más importante y característico: el motor del flujo de trabajo (Workflow Engine). El motor del flujo se encarga de invocar o activar las aplicaciones informáticas que son imprescindibles para efectuar las actividades, además de utilizar los datos referentes a los roles de la organización, control del flujo de trabajo (Workflow Control Data, Workflow Relevant Data) y las listas de actividades (Worklists). Los datos relevantes del flujo de trabajo (Workflow Relevant Data) constituyen los únicos datos a los que puede acceder el motor del flujo de trabajo, siendo generados o actualizados directamente mediante las continuas ejecuciones del los flujos de trabajo.

Las aplicaciones de soporte invocadas durante el proceso (Invoked Applications) se encargan de manipular exclusivamente los datos de las aplicaciones del flujo de trabajo (Workflow Application Data). El motor del flujo de trabajo utiliza estos datos para efectuar transferencias entre las distintas aplicaciones integrantes del proceso que se emplean como soporte de las actividades. Las listas de trabajo (Worklists) son listas donde se colocan las actividades que componen el proceso. Pueden ser visibles o invisibles al usuario, en función de la configuración del sistema. En las visibles, los usuarios acceden directamente a la lista de las actividades para seleccionar aquéllas que van a realizar, pero una tras otra. En las invisibles, es el administrador de la lista (Worklist Handler), un componente software, el que se ocupa de hacer llegar las actividades a los usuarios. La interfaz de usuario (User Interface) es la responsable de materializar la interacción entre usuarios y actividades.

\subsection{Beneficios al automatizar los procesos mediante flujos de trabajo}

La implantación de la tecnología de flujo de trabajo no produce únicamente beneficios sobre los procesos desarrollados en el entorno de oficina, que son numerosos e importantes (Pyke, 1998), sino que además proporciona una serie de notables ventajas en el flujo de documentación del sistema (Hales, 1998):

1. Reducción del uso del papel, ya que la mayoría de los documentos son almacenados y procesados electrónicamente.

2. Solo hay un ejemplar por documento. No hay copias, lo que proporciona más seguridad y menos riesgos.

3. Los documentos no se transfieren físicamente, lo que implica que no se necesita personal dedicado a su distribución. Así, se reduce el coste y tiempo del transporte.

Scire. $9: 2$ (jul.-dic. 2003) 61-83. 
4. Los documentos están siempre disponibles, al encontrarse almacenados en el sistema.

5. Los datos de los documentos pueden ser validados según son introducidos, lo que reduce los errores y retrasos a este respecto.

6. Siempre hay información disponible sobre el progreso de los procesos y sus actividades. El acceso a la información es inmediato.

Todo ello produce una gran repercusión en la organización, pues le proporciona una ventaja competitiva y una mayor eficiencia y efectividad en los procesos que desarrolla. Estos beneficios se sintetizan en la mejora e incremento de la productividad, la mayor calidad en el servicio al cliente y un aumento considerable de las ventas y servicios, al aumentar la eficiencia del proceso.

\section{Fundamentos de la automatización mediante flujos de trabajo}

\subsection{Concordancia entre las necesidades de los entornos documentales y las especificaciones del groupware/workflow}

De los estudios realizados por Sáez (1990) sobre el marco de las oficinas como productoras de información, se extrae un esquema que representa tanto las funciones (trabajo) que se desarrollan en la oficina como las necesidades de automatización que éstas requieren, dando lugar a lo que el autor denomina "modelo conjunto". La estructuración de este modelo se asemeja, en gran parte, a la estructura de tres ejes del groupware, tecnología que, como se ha visto, está orientada

MODELO CONJUNTO

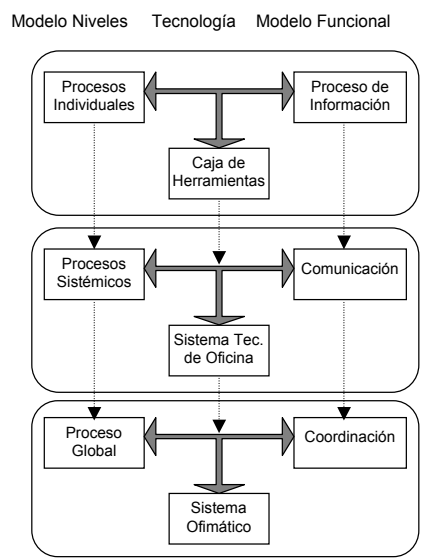

Fig. 5. Concordancia entre el modelo ofimático y las tecnologías groupware (González Lorca, 2001). al soporte del trabajo en grupo. La figura 5 ilustra la confrontación de tales estructuras y las analogías existentes entre ellas. Las funciones de comunicación y colaboración del groupware permiten efectuar a los usuarios y grupos que forman la organización las actividades y funciones encomendadas a su cargo, al darle el soporte tecnológico necesario mediante el software de mensajería electrónica y las bases de datos 
compartidas, entre otros. Así, están capacitados para establecer entre ellos una comunicación efectiva y compartir la información que genera su trabajo.

La complejidad de estos entornos requiere llevar a cabo acciones de coordinación de todos los elementos del sistema, encaminadas a conseguir su total integración y a la eficiencia en el trabajo. Los sistemas de flujo de trabajo materializan esa coordinación, ya que se responsabilizan de establecer un vínculo entre los usuarios, actividades, recursos y reglas que intervienen en los procesos a desarrollar. De esta forma, se establece un único entorno de trabajo, al posibilitar la integración. Además, aporta un carácter dinámico a los procesos, puesto que el sistema de flujo de trabajo distribuye las actividades a los usuarios pertinentes en el momento adecuado junto con los recursos necesarios y las reglas que han de cumplir para desarrollar con garantía de calidad el trabajo asignado. Es verdaderamente importante alcanzar la coordinación de todos los componentes que intervienen en el trabajo, ya que, como pone de manifiesto Sagredo (1996), "la satisfactoria consecución de un proceso empresarial predefinido depende de la coordinación de las personas a la hora de resolver una serie de tareas estructuradas en un orden determinado y dentro de los márgenes temporales previstos". En definitiva, la tecnología de flujo de trabajo permite afrontar las necesidades de automatización de entornos documentales (oficinas), donde la información es su razón de ser, ya que tanto sus características como las de la tecnología que la engloba - el groupware - se ajustan en gran medida al esquema de funcionamiento de una oficina automatizada, o lo que se entiende por automatizar oficinas (González, 2001).

\subsection{Aplicación conjunta del flujo de trabajo y la gestión documental para evitar los flujos caóticos}

Anteriormente se ha expuesto la predisposición que presentan algunas organizaciones para aplicar sistemas de gestión documental en la automatización y mejora de la gestión de sus procesos, y el relativo fracaso que tal iniciativa ha producido, sobre todo por el carácter estático del que adolecen tales herramientas. El planteamiento ideal es la aplicación de la automatización de flujos de trabajo para la gestión de los procesos documentales en los entornos de oficina. Y no es que no exista una clara vinculación entre las tecnologías de flujo de trabajo y de gestión documental, que la hay, ya que ambas se basan, entre otros aspectos, en los documentos y las actividades a las que dan soporte (figura 6). Simplemente se trata de una cuestión de eficiencia.

Los sistemas de gestión documental se emplean con el objetivo de automatizar el tratamiento de la documentación de una organización. Los documentos juegan un doble papel: son el resultado del cumplimiento de las actividades, además de servir de soporte de las mismas. Por otro lado, los sistemas de flujo de trabajo se basan en la automatización de procesos configurados a partir de una serie de

Scire. $9: 2$ (jul.-dic. 2003) 61-83. 


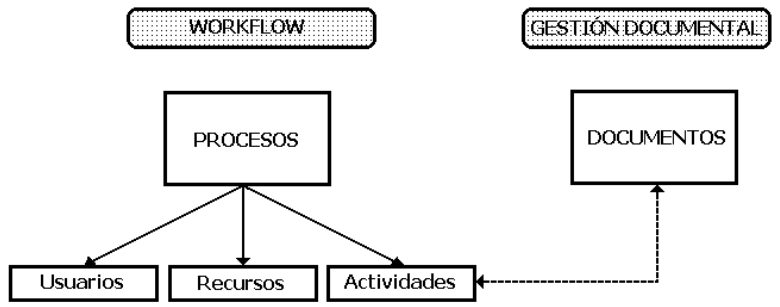

Fig. 6. Los documentos como vínculo entre el flujo de trabajo y la gestión documental. elementos primordiales: usuarios, recursos y actividades. Cada usuario tiene asignadas una serie de actividades y los recursos que necesita para su cumplimiento. Tales actividades también se sustentan en un conjunto de documentos, que informan acerca del procedimiento a seguir en cada una de ellas, y que son el punto de partida para crear otros nuevos. Así, se establece entre ambos software un nexo: los documentos (y las actividades). Los procesos están formados por actividades, que tienen su base en los documentos, los cuales requieren de su tratamiento automatizado y su adecuada gestión para que sirvan eficientemente a los fines de la organización. Para alcanzar un nivel óptimo de gestión de los documentos de la organización es necesario tener en cuenta tanto el flujo de trabajo como el flujo de los documentos. Se trata, en palabras de Hilera (1998), de "asociar un flujo de trabajo o modelo de proceso a cada tipo de documento, con un principio y un final"; es decir, establecer, en torno a una tipología documental, un proceso completo, con sus actividades de inicio y conclusión, procedimientos a seguir, usuarios integrantes y recursos a aplicar, que estarán a disposición de los usuarios para cumplimentar tales actividades.

Se está haciendo referencia a los conceptos de flujo de trabajo y gestión documental como herramientas afines, en términos de integración. Pero no desde el punto de vista de que la gestión documental haya de constituirse como un módulo más en un sistema de flujo de trabajo, engarzado junto al resto de componentes, pues se estaría hablando entonces de una aplicación específica de flujo de trabajo como son los sistemas de gestión integral de expedientes (Calzado, 1996), sino desde la óptica de la complementariedad, pues si por algo se caracterizan los sistemas de flujo de trabajo es precisamente por la independencia existente entre la definición del flujo y las aplicaciones que intervienen en él. El código que forma el flujograma del proceso está totalmente al margen de las aplicaciones que se incluyen en su definición y posteriormente se invocan.

Las aplicaciones que van a formar parte del flujograma, entre las que se encuentran los sistemas de gestión documental (pues son siempre necesarios en un marco de trabajo en el que intervienen documentos), son las encargadas de efectuar las actividades, de gestionar su propio desarrollo; cada actividad requiere una determinada aplicación para su cumplimiento. Los sistemas de 
flujo de trabajo constituyen, por tanto, una herramienta para gestionar la gestión de los procesos; esto es, de meta-gestión, pues en verdad permiten "una nueva configuración y planteamiento de los procesos de la organización, posibilitando la creación de un flujo dinámico en esos procesos y un mayor control y gestión de los mismos, sirviéndose de los recursos disponibles" (González, 2001).

Por último, advertir que la automatización de flujos de trabajo no constituye en sí misma una panacea para la organización. Han de sumarse una serie de factores a ella para dar lugar a la optimización y total eficiencia de los procesos. Esta tecnología facilita el camino a la gestión integral, pero por supuesto se ha de actuar con una cierta lógica dado el contexto en el que se pretende mover la organización, pues si no se procede del modo adecuado, los flujos definidos se convertirán en flujos caóticos, que vienen a ser flujos que, por alguna anomalía concreta en su configuración, se desarrollan perdiendo momentáneamente el control sobre ellos, lo que deriva en una ejecución impredecible e imprecisa temporal o permanentemente.

Un ejemplo claro de flujo caótico lo tenemos en un flujo en el que no se han establecido acciones para implementar la firma digital. De esta forma, cuando sea necesaria la autorización de un superior para efectuar un simple informe, el proceso se detiene, pues se ha de imprimir el documento, colocarse en el portafirmas y esperar a que llegue a dicho superior para que lo firme y otorgue el visto bueno. Si el documento con la firma se digitaliza y se incorpora al proceso, reanudando su ejecución lo antes posible, se trataría de un flujo caótico temporal, ya que el proceso se reanuda posteriormente pero con todos sus elementos mecanizados, mientras que si no se dispone de esta función y el documento permanece en formato papel y separado del resto de documentos del proceso, estamos hablando de un flujo caótico permanente, pues su ejecución ya no es tal y como sería deseable, pues ha de comunicarse al usuario pertinente que se tiene la correspondiente autorización y proseguir con el proceso, faltándole a éste ya un primer documento, que le será incorporado al final, siempre y cuando sea necesario imprimir el expediente para completar su tratamiento.

\section{Estudios de caso en entornos documentales}

La tecnología de automatización de flujos de trabajo no es exclusiva del ámbito de los procesos en una organización comercial, sino que su espectro de aplicación abarca una gran diversidad de entornos, entre los que aquí se destacan los entornos documentales, por ser el objeto del presente estudio. Todo proceso susceptible de ser automatizado mediante una herramienta de diseño de flujos (Definition Tool) es susceptible de funcionar adecuadamente en el entorno tecnológico de los sistemas de flujo de trabajo. A continuación se presentan una serie de flujogramas que muestran la validez de la aplicación de la tecnología de automatización de flujos de trabajo en organizaciones de tipo documental, con-

Scire. $9: 2$ (jul.-dic. 2003) 61-83. 


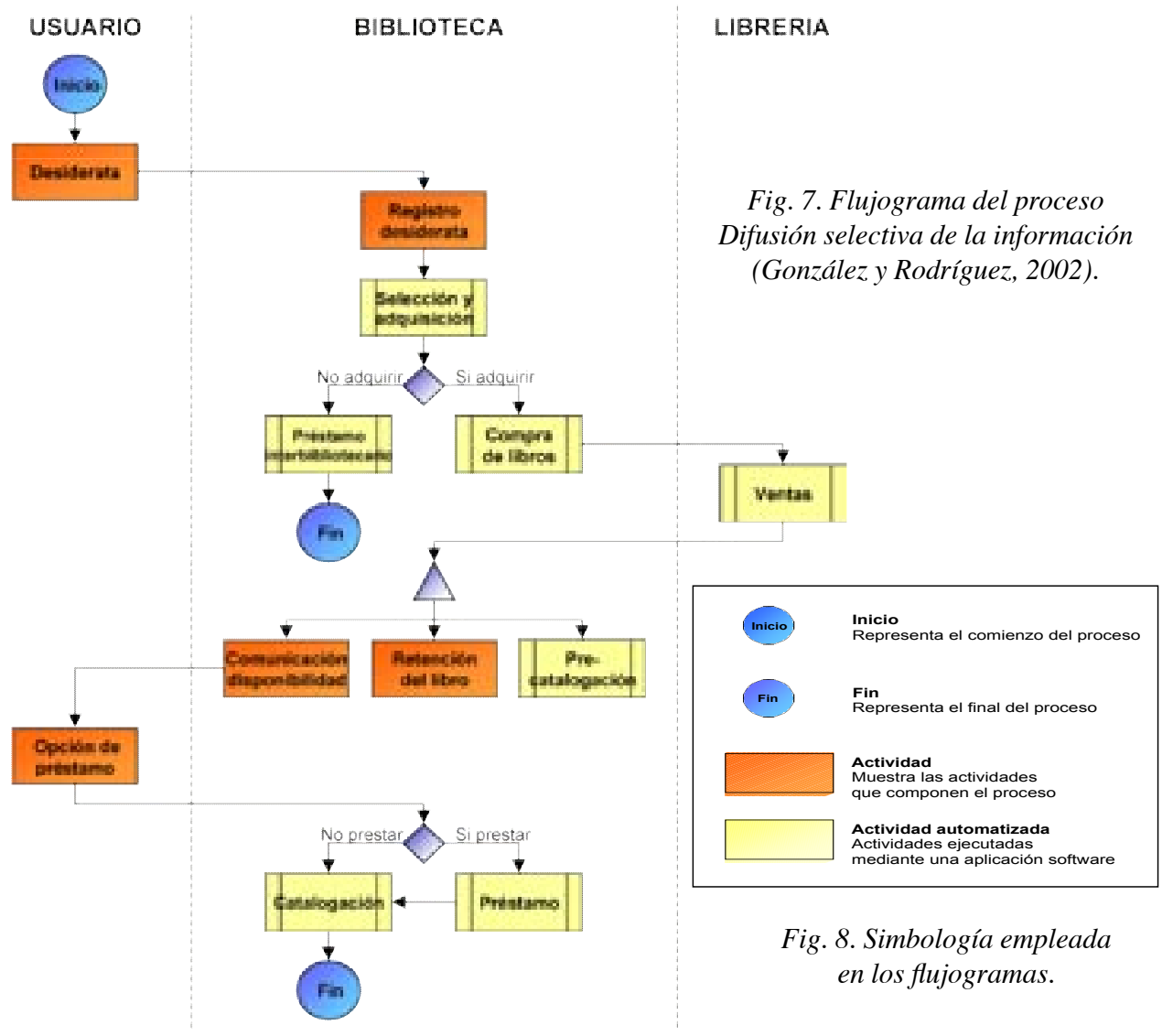

cretamente en archivos, bibliotecas y en el ámbito de la gestión de proyectos, que dan muestra del auge de esta tecnología y su validez para la gestión de procesos de toda índole.

\subsection{Flujo de trabajo en bibliotecas}

Se basa en un trabajo anterior del autor (González y Rodríguez, 2002), en el que se establece un flujograma para un proceso típicamente bibliotecario: $l a$ difusión selectiva de la información (figura 7). El proceso queda formalizado a través de la especificación de las diversas entidades que representan los pasos que integran el proceso, y que se muestran en la simbología utilizada (Figura 8).

\subsection{Flujo de trabajo en archivos}

En un trabajo de Hilera, Gutiérrez y Conde (1999) se plantea la posibilidad de aplicar la tecnología de flujo de trabajo en el entorno de los archivos, concre- 
Automatización dinámica de entornos documentales mediante tecnología workflow

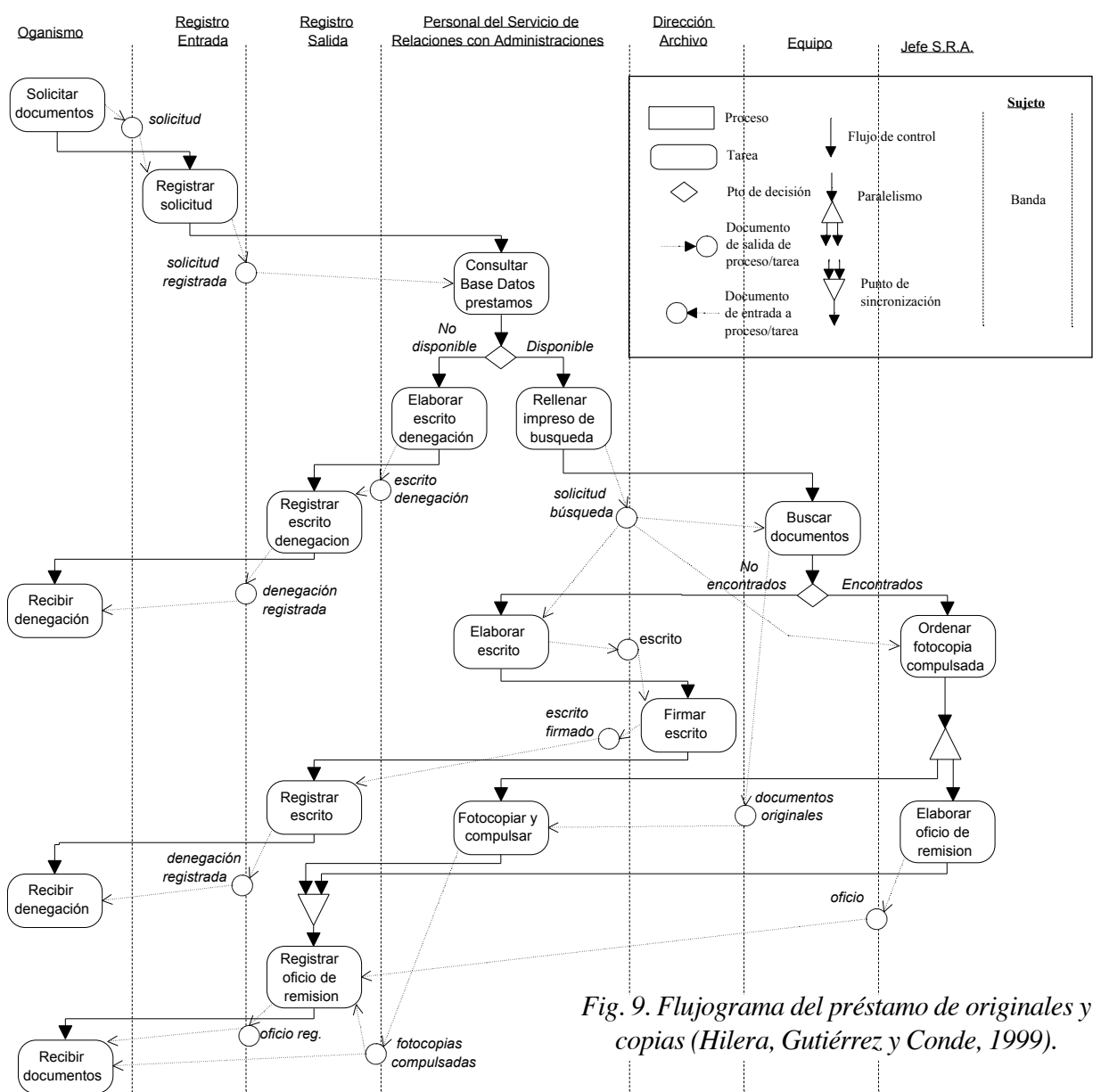

tamente sobre el proceso de préstamo de originales y copia del Archivo General de la Administración Pública. Para ello se valen de una herramienta denominada ToolCADE, desarrollada por el Grupo de Investigación de la Información y Documentación de la Universidad de Alcalá de Henares, con la que definen formalmente el proceso (figura 9). Al lado, en un cuadro se muestra la simbología empleada en el flujograma.

\subsection{Flujo de trabajo en la gestión de proyectos}

Los SAFT son útiles para el control del flujo de documentos. Los ejemplos anteriores así lo corroboran. Pero además también son válidos para automatizar el 
flujo documental de un sistema de gestión de proyectos. A este respecto, el sistema GEDOP (Gedop, 1998), creado a partir de la colaboración entre la Universidad de Oviedo y KYATT Cantábrico, es un sistema de gestión documental orientado al tratamiento de proyectos, que cuenta con módulos específicos para definir y ejecutar flujos de trabajo basados en los documentos que constituyen un proyecto. Éstos sirven a su vez de soporte a la gestión documental y a la gestión de los roles asignados a los participantes (figura 11). En torno a cada tipología documental se crea una estructura con sus elementos (documentos) constituyentes (ficheros Word, Access, etc.), por lo que cada documento que forma parte de un proceso (proyecto) queda totalmente definido (figura 12).

El sistema se ocupa, una vez ejecutado, de enviar los documentos a los roles (usuarios) para que los traten en función de la actividad que han de realizar, desarrollán-

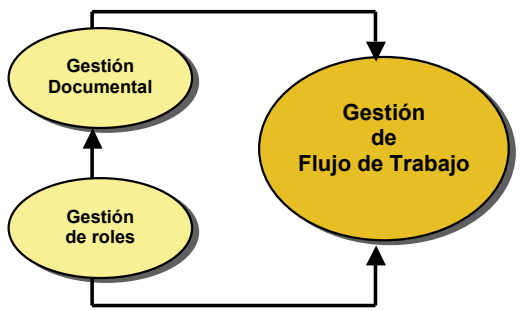

Fig. 11. Estructura lógica del sistema GEDOP (1998).

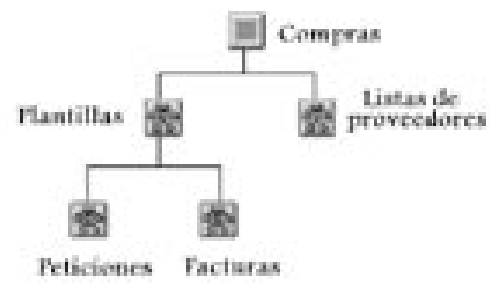

Decranate

Fig. 12. Estructura de un documento en el sistema GEDOP (1998). dose todo el proceso en el marco de un sistema de gestión documental con el apoyo de la automatización de flujos de trabajo, lo que le reporta un claro dinamismo al tratamiento de la documentación en las distintas fases de un proyecto.

\section{Conclusiones}

1. Las organizaciones de tipo documental (entornos documentales) pueden ser consideradas como oficinas, pero desde la óptica de un ente que produce información, que trabaja con información como materia prima esencial.

2. Las oficinas son entornos complejos, cambiantes, pues sufren continuas adaptaciones a sus entornos. Es necesario llevar a cabo acciones de coordinación e integración para garantizar el correcto funcionamiento de sus procesos, la gestión adecuada de los flujos de documentación con los que trabaja y la adaptación a las nuevas necesidades de información.

3. El tratamiento de volúmenes considerables de información, agravado si se encuentra en formato papel, tiene como consecuencia directa una gran dificultad en su gestión, lo que repercute en un desarrollo deficiente de los procesos. Se requiere, por 
tanto, aplicar tecnologías adecuadas que permitan una gestión eficiente de la documentación y, por extensión, también de los procesos en los que interviene.

4. La mayoría de las organizaciones presenta una orgánica funcional, basada en la gestión de funciones, cuando debería funcionar mediante orgánica horizontal, basada en los procesos, para incrementar su eficiencia. Se han aplicado sistemas de gestión documental, pero estas herramientas no son las adecuadas para tal fin, pues su objetivo es el tratamiento automatizado de la documentación, no la gestión integral de procesos.

5. La automatización de flujos de trabajo es una tecnología de coordinación, al permitir la total integración en un mismo entorno de los elementos que intervienen en los procesos de la organización (usuarios-actividades-recursos), y la ejecución dinámica de sus actividades, ya que el sistema se encarga de distribuirlas de forma automática y sucesiva a los usuarios pertinentes junto con los recursos apropiados para efectuarlas. Permite llevar a cabo una gestión integral de procesos. Se trata, por tanto, de una meta-gestión, ya que posibilita establecer flujos dinámicos en los procesos, controlando su ejecución y administración.

6. La aplicación de sistemas de automatización de flujos de trabajo cambia la perspectiva de la organización documental, generando una visión horizontal del trabajo que se centra en los procesos, lo que posibilita la creación de nuevos sistemas de información y beneficia directamente a los productos y servicios que genera.

7. La tecnología de flujo de trabajo produce claros beneficios no únicamente sobre los procesos, sino también sobre el flujo de documentos que les da soporte, lo que origina una mayor eficiencia y efectividad sobre los procesos, produciendo además una ventaja competitiva.

8. Es necesario emplear la tecnología de flujo de trabajo de forma conjunta con la gestión documental para alcanzar un nivel adecuado de gestión de procesos y del flujo de documentos. De esta forma, se puede establecer un tipo de proceso para cada tipología documental, definiendo sus actividades, usuarios y recursos. El sistema se encarga de distribuir estos elementos por el flujo junto con la documentación necesaria en cada caso.

9. La automatización de flujos de trabajo ha de aplicarse con coherencia. No es por sí misma una solución para la organización. Si se pretende automatizar la gestión de un proceso, éste ha de estar completamente automatizado para evitar los flujos caóticos, ya que cualquier paso que no sea definible en el flujograma del proceso provocará irremediablemente una anomalía en el dinamismo de su ejecución. Otra cuestión aparte es si la intención de la organización es aplicar los flujos de trabajo para automatizar parcialmente un proceso, automatizarlo a costa de cualquier acontecimiento o simplemente emplear esta tecnología para estar a la moda.

Scire. $9: 2$ (jul.-dic. 2003) 61-83. 


\section{Notas}

(1) Beca financiada por la Fundación Séneca (Murcia), Centro de Coordinación de la Investigación, a través del programa de Formación del Personal Investigador.

\section{Referencias}

Calzado, V. (1996). De la gestión documental a la gestión integral de expedientes. // Novatica. 120 (marzo-abril 1996) 29-34.

Calzado, V. (1999). El conocimiento como factor competitivo para las empresas. // TICAP: un nuevo mundo de soluciones. Madrid: IDG Communications, 1999. 85-89.

Cleveland, G. (1995). Overview of document management technology. Ottawa: National Library of Canada, 1995. UDT Occasional Paper, 2.

Codina, Ll. (1994). Sistemas de gestión documentales: estado del arte y estrategias de utilización. // Binary. (junio 1994) 114-119.

GEDOP [en línea]. Oviedo: Universidad; KYATT Cantábrico, c1995. 22 diciembre 1998. <http://www.etsimo.uniovi.es/dptos/epm/pi/gedop/gedindex.htm>. Consulta: 3 febrero 1999.

González Lorca, J. (2001). Validez del estudio de la tecnología de flujo de trabajo para la organización automatizada del trabajo corporativo. // Revista Española de Documentación Científica. 24: 2 (2001) 198-211.

González Lorca, J.; Rodríguez Muñoz, J. V. (2002). La tecnología de flujo de trabajo en el contexto de la biblioteca digital. // Anales de Documentación. 5 (2002) 157-175. URL: <http://www. um. es/fccd/anales/ad05/ad0508. pdf>.

Hales, K. (1997) Workflow in context. // Workflow handbook 1997. Chichester: Wiley, 1998. 27-32.

Hilera González, J. R., Gutiérrez De Mesa, J. A.; Conde Villaverde, M. L. (1999). Automatización del flujo de trabajo en unidades de información. Comunicación presentada a congreso científico.

Hilera González, J. R.; Martínez Sánchez, J. M. (1998). El papel de la documentación en la gestión automatizada de flujos de trabajo. // Revista General de Información y Documentación. 8: 2 (1998) 141-147.

Hollingsworth, D. (1995). The Workflow Reference Model [en línea]. Winchester: Workflow Management Coalition, 1995. <http://www. aiim. org/wfmc/standards/ docs/tc003v11. pdf>. Consulta: 1 diciembre 1999.

Johnson-Lenz, P.; T. (1982). Consider the Groupware: design and group process impacts on communication in the electronic medium. New Jersey: Institute of Technology, Computerized Conferencing and Communications Center, 1982. Research report, 16.

Laudon, K. C.; Laudon, J. P. (1997). Administración de los sistemas de información: organización y tecnología. México: Prentice Hall, 1996.

Martínez Sánchez, J. M.; Hilera González, J. R. (1997). Los sistemas de gestión documental en el ámbito del trabajo corporativo. // Revista General de Información y Documentación. 7: 2 (1997) 237-255. 
Pyke, J. (1997). What is workflow enabling? // Workflow handbook 1997. Chichester: Wiley, 1998. 153-156.

Sáez Vacas, F. (1990). Ofimática compleja. Madrid: Fundesco, 1990.

Sagredo, Á. (1996). El workflow como parte del trabajo en grupo. // Novatica. 120 (marzoabril 1996) 37-41.

Saorín Pérez, T. (1997). Ofimática documental. // Comunicación presentada al Congreso de sistemas de información electrónica y nuevos documentos. Zaragoza, febrero de 1997.

Siminiani, M. (1997). Intranets, empresa y gestión documental. Madrid: McGraw-Hill, 1997.

Siminiani, M. (1996). Workflow: qué, para qué y por qué. // Novática. 120 (marzo-abril 1996) 26-28.

Thé, L. (1995). Adentrarse en el flujo de trabajo. // Datamation. 107 (enero 1995) 37-40.

Ticap: un nuevo mundo de soluciones. Madrid: IDG Communications, 1999.

Ultimus (1996). Groupware, workflow and the role of Ultimus [en línea]. [North Carolina]: Ultimus, c1996. 15 May 1998. <http://www. ultimus. com/ultwhite/wp_group. pdf>. Consulta: 25 noviembre 1999.

Vlachantonis, N. (1997). Workflow applications within business organizations. // Workflow handbook 1997. Chichester: Wiley, 1998. 41-48.

Workflow Automation Corporation (2000). Workflow automation [en línea]: new opportunities for dramatic IT results. Ontario (Canadá): Workflow Automation Corporation, c1998. <http://www. workflow. ca/workflow. pdf> [Consulta: 1 junio 2000]. 